\title{
Aging, Reactive Nitrogen Species and Myocardial Apoptosis Induced by Ischemia/Reperfusion Injury
}

\author{
Huirong Liu, Ke Wang, Xiaoliang Wang, \\ Jue Tian, Jianqin Jiao, Kehua Bai, \\ Jie Yang and Haibo $\mathrm{Xu}$ \\ Capital Medical University Beijing \\ P. R. China
}

\section{Introduction}

Globally, the proportion of elderly people is growing faster than any other age group. In 2000 , one in ten, or about 600 million, people were 60 years or older. By 2025, it is expected to reach 1.2 billion people, and in 2050 around 1.9 billion (Hutton, 2008). Aging is a multifactorial process and has been defined as a time-dependent general decline in physiological function of an organism associated with a progressively increasing risk of morbidity and mortality. It is apparent that during aging different organs are losing their functional reserve and plasticity and become less able to fulfill their physiological function, especially under conditions of stress (Beneke \& Bürkle, 2007).

Coronary heart disease is the leading cause of death worldwide, and 3.8 million men and 3.4 million women die of the disease each year (Yellon \& Hausenloy, 2007). Heart aging is accompanied by changes that are progressive, pervasive, potentially deleterious, and, as far as is known, irreversible (Juhaszova et al., 2005). The incidence of heart disease begins to increase in men after the age of forty-five and in women after the age of fifty-five, and the rate for women tends to equal that of men after the age of seventy (MacDonald, 2002). Regardless of age, the heart's well-being is critically dependent on its blood supply, and vascular disease places this in jeopardy. With increasing age, narrowing may develop in the coronary arteries, which lead to the heart. Reduced blood supply causes ischemia (insufficient blood supply for the heart's work) and may produce chest pain or angina. If ischemia is prolonged, irreversible damage resulting in widespread cell death appears, leading to loss of contractility and impairment of cardiac pump function.

Several studies have identified age as a strong predictor of adverse events after acute coronary syndromes; as a result, elderly persons are a high-risk subgroup. Mortality due to ischemic cardiovascular diseases is significantly higher in the elderly than in young adults. The average age for a first MI is 66 years in men and 70 years in women, nearly $50 \%$ of hospital admissions for acute MI and 80\% who die are age 65 years and over (Jugdutt et al., 2008, as cited in Ertl \&Frantz, 2005; Cheitlin \& Zipes, 2001; Thom et al., 2006). After an acute myocardial infarction, early and successful myocardial reperfusion with the use of 
thrombolytic therapy or primary percutaneous coronary intervention (PCI) is the most effective strategy for reducing the size of a myocardial infarct and improving the clinical outcome. The process of restoring blood flow to the ischemic myocardium, however, can induce injury. This phenomenon is termed myocardial ischemia/reperfusion (MI/R) injury. This form of myocardial injury, which by itself can induce cardiomyocyte death and increase infarct size, may in part explain why, despite optimal myocardial reperfusion, the rate of death after an acute myocardial infarction approaches $10 \%$ and the incidence of cardiac failure after an acute myocardial infarction is almost 25\% (Yellon \& Hausenloy, 2007, as cited in Keeley, 2003).

Cell death is a primary factor in the pathogenesis of infarction after $I / R$ and, because survival is strongly correlated to the cardiac myocytes mass lost after $I / R$ injury, can be induced by two different pathways, necrosis and apoptosis which coexist during I/R. Apoptosis is more prevalent than necrosis in senescent animals and humans and increased more significantly in old rats than in young adult ones after renal ischemia reperfusion. Different from necrosis, which is induced by injurious agents, apoptosis is an active programmed cell death characterized by a series of stereotypic morphological and biochemical features. Therefore, it is easier to inhibit apoptosis than to prohibit necrosis for the aging heart. Inhibition of apoptosis may confer protection. So, apoptosis would be one of the efficient targets to ameliorate more serious MI/R injury in aging. In this chapter we focus on aging-induced cardiac myocyte apoptosis and its potential mechanisms after I/ $R$ injury. The chapter is divided into 4 sections: (1)High sensitivity to ischemic heart disease in elderly people; (2) Aging increases myocardial apoptosis under basal and stressed conditions; (3) RNS signaling induced apoptosis contributes to increased susceptibility of aging hearts to myocardial ischemic injury; (4) A mitochondrial pro-apoptotic protein, HtrA2/Omi, is another reason for enhanced MI/R injury in the aging heart.

\section{High sensitivity to ischemic heart disease in the elderly}

\subsection{Risk factors for ischemic heart disease}

Ischemic heart disease (IHD), defined as the earliest manifestation of myocardial infarction, stable or unstable angina pectoris or sudden death, is a vital problem in our society and remains to be a major cause of morbidity and mortality in the elderly. Consequently, the identification of factors related to IHD and quantification of levels of risk is increasingly popular. There are many risk factors contributing to IHD, ranging from blood pressure, both systolic blood pressure (SBP) and diastolic blood pressure (DBP), to living habits like smoking and excessive fat in take. These are undoubtedly the predictors of long term coronary heart disease. IHD, or myocardial ischemic injury, results from severe impairment of the coronary blood supply usually produced by thrombosis or other acute alterations of coronary atherosclerotic plaques resulting from these risk factors.

Curiously, however, the question was raised if these risk factors for IHD found at a young age are still predictive at older ages because IHD may develop in men at a young, middle, or older age. Via long-term studies concerning changing effects of risk factors with age, it has been confirmed (Mathewson et al.,1960; Robert et al.,1998) that blood pressure up to age 65 years manifested itself as a significant factor for IHD - for one thing, the benefit of $10 \mathrm{mmHg}$ lower blood pressure was smaller in older compared to younger men; smoking relevance is 
higher for younger men than for older men; more interesting, the role of either BMI (Body Mass Index) or DM (Diabetes Mellitus) does not vary significantly with age, even though BMI has the largest relative risk in younger men. In 2003, Lakatta and his colleagues ascertained that the aging artery was a "set-up" for vascular diseases (Lakatta \& Levy, 2003). In consideration of studies, both in aging animal models of IHD and censuses on clinical symptoms, it is now well acknowledged that age is a major independent risk factor for IHD although the mechanisms responsible for this age-related pathologic change remain implicit.

\subsection{Aging and IHD}

Aging, characterized by a progressive deterioration of organs and cells, play an imperative role in the manifestation of IHD. The incidence and prevalence of ischemic heart disease increases steeply with advancing age. Considerable evidence from animal studies and clinical observations indicates that aged hearts are more susceptible to I/ $R$ injury, suffer from greater infarct expansion, and exhibit poorer outcomes. It is also disappointing that therapies such as thrombolysis and preconditioning confer less benefit in the aged with myocardial infarction than in younger adults. With the "graying of the population," the morbidity and mortality attributable to cardiovascular diseases will likely continue to increase, resulting in a significant increase in the already huge economic health care burden. These factors call for a multitude of our efforts in uncovering the mechanisms responsible for MI/ $\mathrm{R}$ in aging patients.

\subsection{Possible mechanisms of high sensitivity to IHD in the elderly}

Aging is a complex phenomenon associated with changed DNA transcription and RNA translation together with protein expression. Ischemic heart disease results from severe impairment of the coronary blood supply usually produced by thrombosis or other acute alterations of coronary atherosclerotic plaques. A crucial early step of atherosclerosis is the binding to endothelial cells and subsequent subendothelial migration of circulating monocytes and their transformation into foam cells by the uptake of lipids. Intercellular adhesion molecule-1(ICAM-1) and vascular cell adhesion molecule-1(VCAM-1) in endothelial cells, mediating the adhesion of leukocytes, are suspected of playing an important role in the formulation of early atherosclerotic lesions. Nobuhiro Morisaki (Morisaki et al., 1997) found, through determining the two molecules soluble in serum, that sICAM-1 was correlated only to the presence of IHD but not to age; yet sVCAM-1, not sICAM-1, was positively correlated to age. In other words, age was the most powerful independent predictor of the level of sVCAM-1 and increased susceptibility to ischemia in some sense.

What's more, aging is considered as an energy deficit implicated in myocardial weakness and reduced functions in humans' hearts. The deficit has been demonstrated to be related to the dysfunctional mitochondria that is responsible for the maximum output of ATP, working as a primary site of generation of Reactive Oxygen Species (ROS) and participating in the regulation of apoptosis. With growing age, in the cell and particularly in mitochondria, the defense capacities against ROS become insufficient. Excessive ROS attacks mRNA, DNA, and long-term proteins, which is especially obvious with aging.

A decrease in the total number of myocytes is associated with heart damage and an accelerated decline in cardiac functional capacity observed in aging hearts (Zhang et al., 
2003). It was commonly believed that cardiac myocytes had little ability to regenerate. Therefore, the cardiac function mainly relies on the survival of cardiomyocytes. Two of the most common forms of cell death are programmed cell death and accidental cell death. Programmed cell death is characterized by a series of stereotypic morphological and biochemical features, which is easier to manipulate. It is reported that compared with necrosis, apoptosis is one of the major factors contributing to cardiomyocyte loss. An abundance of evidences certify that cytosolic cytochrome c content is sharply elevated in aging rats (Sharon et al., 2002). Furthermore, Bcl-2, an antiapoptotic protein shows a strong tendency to decrease with age, while Bax, a proapoptotic protein, remains unchanged, so do apoptotic protease-activating factor 1 levels and caspase- 3 activities, regarded as executor caspases following caspase- 8 or caspase- 9 activation. Additionally, chronic oxidative stress with age induces the increase of manganese superoxide dismutase, glutathione peroxidase activity and lipid peroxidation in aging heart mitochondria, which may contribute to IHD. Therefore, age was a major independent risk factor for IHD, and cardiomyocyte death with age may be the last and main event of high sensitivity to IHD.

\section{Aging increases myocardial apoptosis under basal and stressed conditions}

Nowadays, our population is aging at an unprecedented rate, and during 2000-2030, the worldwide population aged $>65$ years is projected to increase by approximately 550 million to 973 million (U.S. Census Bureau), increasing from $6.9 \%$ to $12.0 \%$ worldwide (Kinsella \& Velkoff, 2001). Data from numerous animal researches and clinical studies support that aging is a major independent risk factor in the markedly enhanced risk of cardiovascular diseases in the elderly. There are marked changes related to age in myocardial structure and function in both animals and humans. Previous studies have demonstrated that both diastolic and systolic functions were aggravated in senescent hearts. As the basis of cardiac function, cardiac structure has incurred numerous changes, such as enlarged cardiac myocyte size and reduced myocyte numbers in elderly hearts.

\subsection{Aging and myocardial apoptosis}

Numerous studies have demonstrated that there was a significant loss of cardiac myocytes constituting an important determinant of the aging process, possibly mediating the aggravation of cardiac function. In animal research models, there was around a $32.5 \%$ reduction of myocytes in the left ventricles of rats during a period of 4 to 29 months (Anversa et al., 1990). The study carried out on aging human hearts by G. Olivetti displayed that there was around 35\% aggregate loss of myocytes in both ventricles for 70 year-old healthy men compared to 25 year-old healthy young adults (Olivetti et al., 1991). The factors responsible for the abnormal loss of the cells in the ventricles remain unclear, and it will possibly open a new field to prevent or even reverse the disappearance of cardiac myocytes of aged hearts to reveal the potential mechanism for the aging associated cell loss.

Cell death may occur through active mechanisms like cellular suicide or active or programmed cell death, often referred to as apoptosis. Inactive mechanisms normally induced in acute situations by infarct, severe toxic chemicals and extreme thermal damage, normally conferred as necrosis. Many studies suggest that both apoptosis and necrosis are involved in the aging process (Ohshima, 2006). Apoptosis is more prevalent than necrosis in 
senescent animals and humans and increased more significantly in old rats than in young adult ones after myocardial ischemia reperfusion (Liu et al., 2002). Different from necrosis, which is induced by injurious agents, apoptosis is an active programmed cell death characterized by a series of stereotypic morphological and biochemical features. Therefore, it is easier to inhibit apoptosis than to prohibit necrosis for the aging heart.

\subsection{Apoptosis with age and IHD}

Accumulating studies indicate that aging is associated with an increase in myocardial susceptibility to ischemia and a decrease in postischemic recovery of cardiac function. Zhang et al. reported that in aging rats, myocardial ischemia and reperfusion-induced cardiac injury was enhanced in aging mice as evidenced by enlarged infarct size $(38.5 \pm 2.7 \%$ vs $52.0 \pm 1.8 \%$ ) (Zhang et al, 2007).

As stated above, myocardial apoptosis, a gene-regulated programmed cell death, markedly increases with aging. It has been determined that compared to young mice, myocardial ischemia and reperfusion in aging mice induced an increased apoptosis. Zhang investigated the detailed apoptotic cell death by TUNEL staining and caspase 3 activity assay, and found that TUNEL positive cells increased from $5.0 \pm 1.8 \%$ in young hearts to $8.8 \pm 0.9 \%$ in old ones, and that the caspase 3 activity increased from $18.0 \pm 0.9 \mathrm{nmol} / \mathrm{h} / \mathrm{mg}$ protein to $27.4 \pm 1.9$ $\mathrm{nmol} / \mathrm{h} / \mathrm{mg}$ protein (Zhang et al, 2007). However, the molecular mechanisms and signaling transduction pathways that are responsible for increased susceptibility of cardiomyocytes to apoptosis with aging remain largely unidentified.

\subsection{Signal transduction pathways of apoptosis}

Accumulating evidence indicates that apoptosis is an active, gene-directed process. One of the most widely recognized biochemical features of apoptosis is the activation of a class of cysteine proteases known as caspases. Cells possess multiple caspases that may work in a cascade fashion. The redundancy may serve to amplify and accelerate the response, as well as provide multiple mechanisms for success. Three pathways that activate caspases have been identified: the first one is activated through a cell surface signal leading to caspase 8 (and/or 10 in some cell types) activation; the second, more complicated pathway involves the mitochondria and results in caspase 9 activation; the third is activated via the endoplasmic reticulum and induces caspase 12 activation.

A variety of cellular stresses have been found to cause the disruption and collapse of the inner mitochondrial transmembrane potential, thus opening mitochondrial pores (termed "mitochondrial permeability transition" or MPT) and resulting in the release of cytochrome c. Cytochrome c released from the mitochondria activates caspase 9 in the presence of Apaf1 (apoptosis protease activating factor-1) and ATP. In some cell types, activation of caspase 8 (and/or caspase 2) by the cell surface death receptor also increases mitochondrial cytochrome c release and subsequent caspase 9 activation by facilitating the translocation of full-length or truncated Bid (p15 Bid) from the cytosol to the mitochondrial membrane. As well known, caspase 9 can also be activated by caspase 12 released from the endoplasmic reticulum (ER) under stress. The activation of caspases 8 and 9 (initiator) followed by caspase 3 (executor)(6, and 7 in some cell types) with subsequent degradation of a variety of proteins results in irreversible damage of the cells (apoptotic cell death). Recent studies have 
demonstrated that formation of MPT also results in mitochondrial release of AIF (apoptosisinducing factor), a molecule that directly results in apoptosis.

Under physiologic conditions, apoptosis is regulated by the balance of a variety of pro- and anti-apoptotic molecules. The disturbance of these balances contributes to well-known pathologies, such as neurodegenerative diseases, ischemia/reperfusion injury and cancer. Specifically, the formation of MPT and subsequent release of cytochrome $c$ and AIF are controlled by the balance between anti-apoptotic (e.g., Bcl-2, Bcl-xl, Bcl-w, Bag-1 and BI-1) and pro-apoptotic (e.g., Bax, Bak, Bad, Bid and Bim) Bcl-2 family members, which themselves are regulated by the balance of upstream regulators, such as extracellular signalregulated kinase 1/2 (ERK1/2) and Akt (anti-apoptotic), p38 mitogen-activated protein kinase (MAPK) and JNK (c-Jun N-terminal Kinase) (pro-apoptotic) at both transcriptional and post-transcriptional levels. In a recent study, Jiang and colleagues reported that activation of caspase 9 is positively regulated by tumor suppressor putative HLA-DRassociated proteins (PHAP) but inhibited by oncoprotein prothymosin- $\alpha$ (ProT)(Jiang et al, 2003). Finally, the activation of caspase 3 is inhibited by IAP (inhibitor of apoptosis protein) and $\alpha \mathrm{B}-\mathrm{Crystallin}$, and promoted by Smac/DIABLO and Omi/HtrA2 (proteins that are also released from mitochondria under stress) by interaction with IAP. Given the extreme complexity of the apoptosis signal transduction and the exponentially-increasing number of pro- and anti-apoptotic molecules, it is highly unlikely that a specific cell type, i.e., cardiomyocytes, under specific stress, i.e., aging, will involve all of these apoptotic molecules and disturb every pro- and anti-apoptotic balance. Identifying the specific anti/pro-apoptotic balance(s) that is (are) disturbed by aging, and uncovering the mechanisms responsible for the deregulation of apoptotic cell death in the aging heart will be the critical first step toward prevention and treatment of apoptotic cell death in patients with any heart disease. In the next section we will identify the reactive nitrogen species (RNS) signal and HtrA2/Omi related mitochondrial apoptotic pathway disturbed in the aging heart.

\section{RNS signaling induced apoptosis contributes to increased susceptibility of aging hearts to myocardial ischemic Injury.}

\subsection{An overview of reactive nitrogen species and pathway}

Considerable evidence suggests that reactive oxygen species (ROS) play critical roles in the aging process and post-ischemic myocardial apoptosis. However, the outcome of clinical trials with antioxidant treatment during MI/R has been rather disappointing, suggesting that other factors in addition to ROS exist. Most recent data have identified nitric oxide derived reactive nitrogen species (RNS) intermediates as critical contributors and cell injury.

RNS are produced by the reaction of NO with superoxide (Carr et al., 2000) and include NO and its derivatives (Wu et al., 2007). NO, as the most important mediator of RNS, has been identified as having three categories of signaling pathways in controlling cell death: (1) Classical signaling involves the selective activation of soluble guanylate cyclase (sGC) (cGMP-dependent protein kinases) (2) Less classical signaling: NO binds to cytochrome $\mathrm{c}$ oxidase $(\mathrm{CcO})$ in the mitochondria and its functional consequences (3) Nonclassical signaling alludes to the formation of NO-induced posttranslational modifications (PTMs), especially S-nitrosylation, S-glutathionylation, and tyrosine nitration(Antonio et al., 2011). 


\subsubsection{Nitric oxide signaling pathway - Classical signaling pathways and apoptosis}

In classical signaling pathways, NO induces the rupture of the His-Fe(II) bond within the heme of sGC, which catalyzes in the conversion of GTP to cGMP (Antonio et al., 2011). Effects of cGMP occur through three main groups of cellular targets: cGMP-dependent protein kinases (PKGs), cGMP-gated cation channels, and PDEs. cGMP binding activates PKG, which phosphorylates serines and threonines on many cellular proteins, frequently resulting in changes in activity or function, subcellular localization, or regulatory features (Francis et al., 2010). Accumulating data have showed NO could regulate apoptosis in classical signal pathways.

\subsubsection{1 cGMP-Dependent inhibition of apoptotic signal transduction}

Research has shown that NO protects against PC12 cell death by inhibiting the activation of caspase proteases through cGMP production and activation of protein kinase G (Kim et al., 1999). Another work further proves that the NO/cGMP pathway suppresses 6-OHDAinduced PC12 cell apoptosis by suppressing the mitochondrial apoptosis signal viaPKG/PI3K/Akt-dependent Bad phosphorylation (HA et al., 2003). NO has also been shown to inhibit TNF-a-induced TRADD recruitment and caspase 8 activity in a cGMPdependent way (De Nadai et al., 2000). More direct data shows that over production of endogenous NO or supplementation with exogenous NO protects SK-N-BE human neuroblastoma againstapoptosis induced by serum deprivation by using cGMP as an intermediate effector (Ciani et al., 2002). The anti-apoptotic role of NO through cGMP is also revealed in hepatocytes (Calafell et al., 2009). In vivo data reveal that enhancing the $\mathrm{NO} / \mathrm{cGMP}$ pathway reduces hypoxia-induced neuron apoptosis by upregulating the bcl2/Bax ratio (Caretti et al., 2008). NO has also been shown to antagonize estrogen induced apoptosis in breast epithelial cells (Kastrati et al., 2010).

\subsubsection{2 cGMP-Dependent induction of apoptotic signal transduction}

Despite evidence that NO is anti-apoptotic, data also show that NO can induce apoptosis in a cGMP dependent manner in isolated adult cardiomyocytes. Induction of apoptosis by SNAP in cardiomyocytes was blocked by inhibitors of caspase-3, soluble guanylyl cyclase (sGC) or of cGMP dependent protein kinase (PKG) (Taimor et al., 2000). In vascular cells NO is shown to induce apoptosis through cGMP-dependent Protein Kinase (PKG) (Chiche et al., 1998).

\subsubsection{Nitric oxide signaling pathway - Less classical signaling pathways and apoptosis}

The critical mediator of NO regulating apoptosis in less classical signalling pathways is cytochrome c oxidase $(\mathrm{CcO})$, which is located on the inner membrane of the mitochondrion and catalyzes the oxidation of cytochrome $\mathrm{c}$ and the reduction of oxygen to water (Taylor \& Moncada, 2010). The enzyme belongs to the superfamily of heme-copper oxidases and contains a highly conserved bimetallic active site composed of the heme iron of cytochrome a3 and a copper ion, CuB. The binuclear center, in its reduced form, is the binding site of the physiological substrate, $\mathrm{O} 2$. NO closely resembles $\mathrm{O} 2$ and therefore can also bind to this site (Martinez-Ruiz et al., 2011). NO at low concentrations inhibits mitochondrial $\mathrm{CcO}$, resulting in an increase in $\mathrm{H} 2 \mathrm{O} 2$ in the mitochondria, and this excess of $\mathrm{H} 2 \mathrm{O} 2$ activates the death signaling pathways (Yuyama et al., 2003). CcO potentially generates impairment of 
mitochondrial function, which in turn may potentiate apoptotic commitment. The NO toxicity can be indicated by the decrease of cytochrome c oxidase activity, which is paralleled the extent of apoptosis (Ciriolo et al., 2000).

\subsubsection{Nitric oxide signaling pathway-nonclassical signaling pathways and apoptosis}

Nitric oxide could exert part of its effects not only in a cGMP independent manner, but also without the need of binding to other metal centers, through covalent posttranslational modification (PTM) of target proteins. This mode of action is defined as denominate nonclassical signaling and affects mainly cysteine and tyrosine residues in proteins. The three best known NO-induced posttranslational modifications are S-nitrosylation, Sglutathionylation, and tyrosine nitration.

\subsubsection{S-nitrosylation and apoptosis}

S-nitrosylation is an important biological reaction of nitric oxide; it refers to the conversion of thiol groups, including cysteine residues in proteins, to form S-nitrosothiols. SNitrosylation is a mechanism for dynamic, post-translational regulation of most classes of protein. NO has been shown to regulate apoptosis through S-nitrosylation of protein.

Research hasrevealed that S-nitrosylation mainly antangonizes apoptosis by targeting many elements in both extrinsic and intrinsic pathways (Iyer et al., 2008). Fas, as a cell surface receptor, is a member of the tumor necrosis receptor superfamily that induces apoptosis when cross-linked by Fas ligand or by Fas agonist antibody (Park et al., 2006; Lavrik et al., 2005; Mannick \& Schonhoff, 2004). Regulation of cell signaling by protein nitrosylation is well exemplified in the Fas signalling pathway (Mannick \& Schonhoff, 2004). Consistent with receptor-mediated apoptosis, two main pathways of Fas-mediated apoptosis have been identified (Park et al., 2006). In type1 cells caspase- 8 directly cleaves caspase-3, which starts the death cascade. In type 2 cells the quantity of caspase- 8 is insufficient to directly activate the executioner caspase-3. Instead, it involves (activates) tBid- mediated cytochrome c (CytoC) release from mitochondria followed by apoptosome formation (Park et al., 2006; Lavrik et al., 2005). In resting cells caspase-3 zymogens in mitochondria are kept inactive via Snitrosylation of their catalytic site cysteine. Caspase-3 may be S-nitrosylated in mitochondria due to an association between S-nitrosylated caspase-3 and NOS. Moreover, S-nitrosylated but not denitrosylated caspase-3 associates with acid sphingomyelinase (ASM) in mitochondria. The association of S-nitrosylated caspase-3 with ASM provides another level of apoptosis regulation by inhibiting capase- 3 cleavage and activation by initiator caspases. When cells are stimulated by Fas ligand, caspase- 3 becomes denitrosylated. Denitrosylation stimulates caspase- 3 activity by two mechanisms. First, denitrosylation allows the catalytic site of caspase- 3 to function. In addition, denitrosylated caspase-3 presumably dissociates from ASM, allowing initiator caspases to cleave caspase-3 to its fully active form. Thus Snitrosylation/denitrosylation serves as an off/on switch for caspase-3 function during apoptosis. Cyto-C activity is also regulated by nitrosylation during Fas-induced apoptosis. However, in contrast to caspase- 3, Cyto- $C$ is not nitrosylated in resting cells. Instead, when cells receive an apoptotic stimulus, Cyto- $C$ is nitrosylated on its heme iron in mitochondria and then is rapidly released into the cytoplasm. In the cytoplasm, hemenitrosylated Cyto-C stimulates caspase- 3 cleavage by the apoptosome. Thus, coordinated denitrosylation of caspase- 3 and hemenitrosylation of Cyto- $C$ serves to enhance caspase activation and Fasinduced apoptosis. It remains to be determined if denitrosylation of caspase- 3 is directly 
linked to nitrosylation of Cyto-C in mitochondria via a direct transfer of a NO+ group from the catalytic site cysteine of caspase-3 to the heme iron of Cyto-C (Mannick et al., 1997; Mannick \& Schonhoff, 2004; Schonhoff et al., 2003; Stamler et al., 2001(Mannick et al., 1997; Mannick \& Schonhoff, 2004; Schonhoff et al., 2003; Stamler et al., 2001). NO can also inhibit apoptosis by direct nitrosylation of caspase-9 ( Török et al., 2002).

In intrinsic apoptosis pathways, Cyto-C is released from mitochondria into cytoplasm initiates the apoptotic signals (Brüne, 2003; Schonhoff et al., 2003) and has been suggested as the commitment step for apoptosis (Gaston et al., 2003). Previous studies suggest that nitrosylation of Cyto-C is a novel mechanism of apoptosis regulation in cells and a very early event in apoptotic signalling (Schonhoff et al., 2003). However, the critical commitment step in the mitochondrial pathway of apoptosis has not been firmly established. Several recent findings suggest that caspase- 9 activation is essential for, and likely represents, the commitment step for the mitochondrial pathway of apoptosis. Nitrosylation of caspase- 9 by induced (i) NOS generated NO inhibits apoptosis downstream of Cyto-C release and would appear to be another mechanism negatively regulating this pathway of apoptosis (Török et al., 2002). Besides nitrosylation of caspases, another mechanism underlying the antiapoptotic effects of NO via S-nitrosylation includes stimulation of the anti-apoptotic activity (function) of thioredoxin (Trx), which depends on S-nitrosylation at Cys69 (Haendeler et al., 2002). S-nitrosylation and inhibition of Apoptosis signal regulating kinase (ASK1) (in L929 cells) at Cys869 also lead to anti-apoptosis (Park et al., 2004).

Although most of the reports have proven that S-nitrosylation mainly inhibits apoptosis, there are also data showing that S-nitrosylation could induce apoptosis as well. The mechanisms underlying the pro-apoptotic effects of $\mathrm{NO}$ via S-nitrosylation include inhibition of the anti-apoptotic transcription factor NF- $\kappa B$ through a variety of mechanisms, including S-nitrosylation of NF- $\mathrm{KB}$ (in A549 cells) or nitrosylation of the target cysteine in the IкB kinase complex (IKK) (in Jurkat cells) leading to decreased NF- $\kappa B-m e d i a t e d$ transcription and decreased Bcl-2 expression (Marshall \& Stamler, 2002; Schonhoff et al., 2006). p21ras, JNK kinase, and the p50 monomer (of p50-p65) have been identified as sites of S-nitrosylation that mediate the stimulation or inhibition of NF- $\kappa B$ by NO (Marshall and Stamler, 2002). Glyceraldehyde-3-phosphate dehydrogenase (GAPDH) is S-nitrosylated by $\mathrm{NO}$, which initiates an interaction with the E3 ligase Siah1, leading to nuclear translocation and ubiquitin-mediated degradation of nuclear target proteins (Benhar \& Stamler, 2005). Hara et al. has demonstrated that deprenyl and TCH346 are neuroprotective by preventing the S-nitrosylation of GAPDH and inhibiting GAPDH/Siah cell death cascade (Hara et al., 2006). NO can also enhance apoptosis by NO-induced persistent inhibition and nitrosylation of mitochondrial Cyto-C oxidase in lung endothelial cells (Zhang et al., 2005). The work done by $\mathrm{Gu}$ et al. has illustrated that S-Nitrosylation activated Matrix metalloproteinase-9 in vitro was implicated in the pathogenesis of neurodegenerative diseases, stroke, and induced neuronal apoptosis (Gu et al., 2002).

\subsubsection{S-Glutathionylation and apoptosis}

S-thiolation refers to the incorporation of a low-molecular-mass (LMM) thiol to a protein via formation of a mixed disulfide bridge between a cysteine residue and the LMM thiol. In the intracellular environment in which GSH is the major thiol present, its incorporation results in a PTM named S-glutathionylation or S-glutathiolation, or more commonly S-thiolation 
(Martinez-Ruiz et al., 2011). Protein S-glutathiolation, the reversible covalent addition of glutathione to cysteine residues on target proteins, is emerging as a candidate mechanism by which both changes in the intracellular redox state and the generation of reactive oxygen and nitrogen species may be transduced into a functional response (Klatt \& Lamas, 2000). Sglutathionylation is a redox signaling mechanism that can be produced without the concourse of NO. However, evidence for S-glutathionylation induced by NO and/or RNS has accumulated, linking this modification with NO signalling (Giustarini et al., 2004). At least two mechanisms explain the link between RNS production and S-glutathionylation. One is the observed glutathionylation induced by peroxynitrite. The other is a nitrosylated protein cysteine may react with GSH, or S-nitrosoglutathione can be formed and react with the cysteine thiol, both leading to S-glutathionylation (Martinez-Ruiz et al., 2011).

Vikas Anathy et al. demonstrated that stimulation with Fas ligand (FasL) induces Sglutathionylation of Fas at cysteine 294 independently of nicotinamide adenine dinucleotide phosphate reduced oxidase - induced ROS. Instead, Fas is S-glutathionylated after caspasedependent degradation of Grx1, increasing subsequent caspase activation and apoptosis (Anathy et al., 2009). Suparna Qanungo et al. indicated S-glutathionylation of p65-NFkB as a major mechanism underlying the inhibition of the NF_B survival pathway and promotion of apoptosis after GSH supplementation in hypoxic pancreatic cancer cells (Qanungo et al., 2007). Therefore, RNS could regulate apoptosis through S-glutathionylation of protein

\subsubsection{Tyrosine nitration and apoptosis}

RNS-mediated nitration modifications include nitration of tyrosine, tryptophan, amine, carboxylic acid, and phenylalanine groups. However, nitration of tyrosine residues to produce nitrotyrosine has recently received much attention. Protein tyrosine nitration is a covalent protein modification resulting from the addition of a nitro (-NO2) group onto one of the two equivalent ortho carbons of the aromatic ring of tyrosine residues (Gow et al., 2004). Biological nitration of tyrosine depends largely on free radical chemistry. There are two main key nitration pathways that operate in vivo and involve peroxynitrite and hemoperoxidase-catalyzed nitration (Peluffo \& Radi, 2007). Tyrosine nitration is a two-step process where the initial reaction is the oxidation of the aromatic ring of tyrosine to yield tyrosyl radical $(\mathrm{Tyr} \bullet$ ) (oxidation step), which in turn adds $\bullet$ NO2 (addition step) to yield 3NO2-Tyr (Peluffo \& Radi, 2007).

\subsection{RNS-mediated Tyrosine nitration induction of Apoptotic Signal Transduction}

Work by Hortelano et al. indicates that nitric oxide-dependent apoptosis in macrophages occurs in the presence of a sustained increase of the mitochondrial transmembrane potential, and that the chemical modification and release of cytochrome $\mathrm{c}$ from the mitochondria precedes the changes of the mitochondrial transmembrane potential. NO-dependent apoptosis in macrophages involves a chemical modification of cytochrome $\mathrm{c}$ that alters its structure and facilitates release from the mitochondria, regardless of the changes of the mitochondrial transmembrane potential (Hortelano et al., 1999). Cassina et al. has shown that Tyr-67 is a preferential site of nitration among the four conserved tyrosine residues in cytochrome c. Cytochrome c3+ was more extensively nitrated than cytochrome c2+ by mitochondrial but also cytosolic or extracellular derived ONOO- diffusing to the intermembrane space (Cassina et al., 2000). Tao et al. proved that nitrative inactivation of Trx plays a proapoptotic role in postischemic myocardium (Tao et al., 2006). Studies 
conducted by Li et al. demonstrated that there exists a TNF-a-initiated, cardiomyocyte iNOS/NADPH oxidase-dependent, peroxynitrite-mediated signaling pathway that contributes to posttraumatic myocardial apoptosis. In this paper nitrotyrosine content acted as a footprint of in vivo peroxynitrite formation (Li et al., 2007).

\subsection{RNS-Mediated tyrosine nitration inhibition of apoptotic signal transduction}

Although many studies have shown that RNS-mediated tyrosine nitration mainly induce apoptosis. Some research indicated that it also can inhibit apoptosis. Work by Sonsoles Reinehr et al. indicates that CD95 nitration as a novel mechanism for apoptosis inhibition by NO, which competes with pro-apoptotic CD95-tyrosine phosphorylation(Reinehr et al,2004). The study of Nakagawa et al determined that cytochrome $\mathrm{c}$ nitrated by continuous treatment with peroxynitrite lost its ability to cause caspase cascade activation in vitro, whereas cytochrome $\mathrm{c}$ nitrated by a bolus peroxynitrite treatment had preserved activity (Nakagawa et al, 2007).

Previous data have shown that Reactive Nitrogen Species can either stimulate (proapoptosis) or prevent apoptosis (anti-apoptosis) (Boyd \& Cadenas, 2002; Brüne, 2003; Choi et al., 2002; Patel et al., 1999). The concentrations and local environments including cellular redox state and the presence of free radicals of NO and RNS play a key role in determining whether they stimulate or inhibit apoptosis (Brüne, 2003). Peroxynitrite(ONOO-), an important RNS, is formed by the reaction between high concentrations of $\mathrm{NO}$ and superoxide . High concentrations of $\mathrm{NO}$ or ONOO-can induce apoptosis (Choi et al., 2002). Liang et al. demonstrated for the first time that L-arginine administered at different time points during I/R exerted different effects on post-ischemic myocardial injury and suggests that stimulation of eNOS reduces nitrative stress and decreases apoptosis whereas stimulation of iNOS increases nitrative stress and enhances myocardial reperfusion injury (Liang et al., 2004). But, Rus et al.'s results demonstrated that inhibition of iNOS raises the peroxidative and apoptotic level in the hypoxic heart indicating that this isoform may have a protective effect on this organ against hypoxia/reoxygenation injuries, and this challenges the conventional wisdom that iNOS is deleterious under these conditions (Rus et al., 2010). So, the effect on apoptosis of RNS and its regulation need further clarification.

\subsection{RNS signaling and aging myocardial ischemic injury}

Accumulated data have shown that nitric oxide derived reactive nitrogen intermediates are critical contributors in controlling apoptosis which determine the susceptibility of aging hearts to myocardial ischemic Injury.

Gao et al's results showed that the protective effects of adenosine on myocardial I/R injury are markedly diminished in aged animals and that the loss in NO release in response to adenosine may be at least partially responsible for this age-related alteration (Gao et al., 2000). Studies also show that increased susceptibility of the type 2 diabetic GK rat heart to ischemic injury is not associated with impaired energy metabolism. Reduced coronary flow, upregulation of eNOS expression, and increased total NOx levels confirm NO pathway modifications in this model, presumably related to increased oxidative stress. Modifications in the NO pathway may play a major role in I/R injury of the type 2 diabetic GK rat heart (Desrois et al., 2010). Our results show that aging induces phenotypic upregulation of iNOS in the heart, in which $\beta$-AR stimulation interacts with ischemia and triggers a markedly 
increased NO production, which creates a nitrative stress, generates toxic peroxynitrite, activates apoptosis, and eventually causes cardiac dysfunction and myocardial injury. An iNOS inhibitor-1400W can markedly attenuate these adverse effects in the aging heart (Li, 2006).

\subsection{Thioredoxin and aging-related myocardial apoptosis}

Thioredoxin (Trx) is a 12-kDa protein ubiquitously expressed in all living cells that fulfils a variety of biological functions related to cell proliferation and apoptosis. It is involved not only in cytoprotective functions against oxidative stress but also in the regulation of cellular proliferation and the aging process. Clinical and experimental results have demonstrated that inhibition of Trx promotes apoptosis (Lincoln et al, 2003). Recent in vitro studies demonstrate that $\operatorname{Tr} x$ interacts directly with, and inhibits, the activity of apoptosisregulating kinase-1 (ASK1), a mitogen-activated protein (MAP) kinase that activates two proapoptotic kinases, p38 MAP kinase (MAPK) and c-Jun N-terminal kinase (JNK) (Liu \& Min, 2002). In aged mouse livers, the ratio of ASK1/Trx-ASK1 (free ASK1/Trx-binding ASK1) increases and this correlates with the increased basal activity of the p38 MAPK pathway. These results suggest that Trx may play critical roles in cell proliferation and cell death in aging, and Trx activity/expression might be reduced in the aging heart, thus tilting the death/survival balance toward cell death and promoting ischemia/reperfusion injury.

Under physiologic conditions, ASK1 activity is inhibited by several cellular factors, including Trx, glutaredoxin, and phosphoserine-binding protein 14-3-3 (Bishopric \& Webster, 2002). Previous studies have demonstrated that many cellular stresses and apoptotic stimuli activate mitochondrial-dependent apoptotic pathways by facilitating dissociation of ASK1 with its inhibitory protein. Trx is physically associated with ASK1 in cardiac tissues from young animals. However, Trx-ASK1 binding was reduced in cardiac tissue from aging animals. Therefore, it is likely that increased posttranslational Trx modification in aging hearts results in disassociation of Trx from ASK1, thus increasing postischemic myocardial apoptosis by increasing p38 MAPK activity.

Lots of studies have demonstrated that, in addition to upregulation or downregulation of Trx expression at the gene level, Trx activity is regulated by posttranslational modification. Three forms of posttranslational modification of Trx have been previously identified. These include oxidation, glutathionylation, and S-nitrosylation. Interestingly, all three forms of modification occur at cysteine residues but affect Trx function differently. Oxidation of the thiol groups of Cys-32 and-35 forms a disulfide bond which results in Trx inactivation. However, previous studies have demonstrated that administration of oxidized Trx- 1 exerts significant antioxidant and cytoprotective effects unless intracellular Trx reductase is inhibited, indicating that oxidative $\operatorname{Trx}$ inhibition is reversible and this form of posttranslational modification may not be the major mechanism responsible for Trx inactivation in vivo (Andoh et al, 2003). Glutathionylation occurs at Cys-73, and this posttranslational modification significantly inhibits Trx activity (Casagrande et al, 2002). However, whether Trx glutathionylation may occur in vivo in diseased tissues remains completely unknown and the role of this form of posttranslational modification in regulating Trx function in vivo remains to be determined. S-nitrosylation has been reported to occur at either Cys-69 or Cys-73. In contrast to oxidation and glutathionylation, Snitrosylation increases Trx activity and further enhances its antiapoptotic effect (Haendeler et al., 2004; Mitchell \& Marletta, 2005). 
In a recent study, it has been demonstrated that, in addition to three previously reported posttranslational Trx modifications which all occur at the cysteine residue, Trx can also be modified at the tyrosine residue (protein nitration) in a peroxynitrite-dependent fashion (Tao et al., 2006). More interestingly, in contrast to the reversible (by Trx reductase) oxidative $\operatorname{Trx}$ inactivation, nitrative modification of $\operatorname{Trx}$ results in an irreversible inactivation. Therefore, nitric oxide and its secondary reaction products, particularly peroxynitrite, exert opposite effects on Trx activity. Specifically, nitric oxide itself induces Trx S-nitrosylation and enhances its activity. In contrast, peroxynitrite results in Trx nitration and causes an irreversible inactivation. In Zhang et al.'s study, Trx activity was determined by using the insulin disulfide reduction assay. Compared with young animals, cardiac Trx activity is decreased in the aging heart before myocardial ischemia and reperfusion, and this difference can be further amplified after myocardial ischemia and reperfusion. However, Trx expression is slightly increased, rather than decreased, in aging hearts. These results indicate that it is posttranslational Trx modification rather than reduced protein expression that reduces Trx activity in the aging heart (Zhang et al., 2007).

\section{A Mitochondrial pro-apoptotic protein, HtrA2/Omi, is another reason of enhanced $M I / R$ injury in the aging heart}

It is well known that apoptotic cell death is orchestrated by a family of caspases. Xchromosome linked inhibitor of apoptosis protein (XIAP), as a member of IAPs, was the most potent endogenous inhibitor of caspases in human beings. XIAP has three baculovirus IAP repeat (BIR1, BIR2, BIR3) domains and a really interesting new gene (RING) domain. Biochemical studies suggested BIR2 inhibits caspase-3 and caspase-7, whereas BIR3 inhibits caspase-9 (Deveraux et al., 1999). The RING domain is an E3 ligase that presumably directs targets to the ubiquitin-proteasome degradation system, such as caspase-3 (Salvesen \& Duckett, 2002; Martin, 2002). The anti-apoptotic activity of XIAP is regulated by a group of proteins that bind to the BIR domains via N-terminal conserved 4-residue IAP-binding motif (Shi, 2002). Recently it has been shown that overexpression of XIAP via in vivo delivery in an adenovirus could reduce both myocardial apoptosis and infarction following I/ R (Kim et al., 2011). Wang et al. the protein and mRNA content of XIAP in the heart after MI/R was decreased, while the protein content of XIAP showed positive correlation with cardiac function in 42 rats after MI/R(Wang et al., 2010). These findings suggested a link between myocardial apoptosis, and anti-apoptotic therapy was effective in reducing $I / R$ injury. Meanwhile we found the degradation of XIAP in aging myocardium after MI/R was more than that in young myocardium after MI/R, which are consistent with previous results in which myocardial apoptosis was exaggerated with aging after MI/R. Additionally, the expression of XIAP was also significantly decreased than that in the young adult heart without the intervention of MI/R, which suggested that the decline of XIAP expression may be a major factor responsible for the increased susceptibility of the aging heart.

$\mathrm{XIAP}$ is regulated by two cellular proteins, Smac/DIABLO and HtrA2/Omi, which are nuclear-encoded mitochondrial proteins. The cleavage of their mitochondrial-targeting sequences inside mitochondria generates processed active Smac /DIABLO and HtrA2/Omi with new apoptotic N termini, named the IAP-binding motif (IBM) (Srinivasula et al., 2003). Stimulated by apoptotic triggers, Smac/DIABLO and HtrA2/Omi release into the cytosol and competitively bind to the BIR domains of IAPs via IBM, so that the BIR-bound caspases 
are released and reactivated, resulting in cell apoptosis (Wu et al., 2000, Suzuki et al., 2001). Unlike Smac/DIABLO, the pro-apoptotic activity of HtrA2/Omi involves not only IAP binding but also serine protease activity. Although Omi/HtrA2 and Smac/DIABLO both seem to target XIAP once released into the cytosol, increasing evidence suggests that Omi/HtrA2 may play a unique role in apoptosis. Several different Smac/DIABLO-deficient cells respond normally to various apoptotic stimuli, suggesting the existence of a redundant molecule or molecules compensating for a loss of Smac/DIABLO function (Okada et al., 2002). In contrast, Omi/HtrA2-knockdown cells have shown to be more resistant to apoptotic stimuli (Martins et al., 2002).

In addition, Liu et al. first provided direct evidence that a normal level of endogenously expressed HtrA2/Omi contributes to apoptosis after MI/R in vivo (Liu et al., 2005). Althaus et al. have also suggested that HtrA2/Omi plays a decisive role in apoptosis after MI/R in young rats (Althaus et al., 2007). Then Wang et al showed that the release of HtrA2/Omi from mitochondria to cytosol was significantly increased in the old MI/R rat heart compared with that in the young MI/R rats (Wang et al, 2006). Meanwhile, cytosol was markedly increased in the old sham group compared with that in the young sham group. Taken together, these results reveal that HtrA2/Omi plays a causative role in increased post-ischemic cardiomyocyte apoptosis in the aging heart (Okada et al., 2002). In order to investigate whether increased HtrA2/Omi plays an important role in aged myocardial apoptosis resulting in myocardial dysfunction and increased susceptibility to MI/R injury, Wang et al observed the effect of ucf-101, a highly selective Omi/HtrA2 inhibitor, on MI/R injury. They have provided direct evidence in the current study that treatment with ucf-101 in aging MI/R animals reduced the caspase-3 activity and improved the cardiac functions. Their results demonstrated that translocation of Omi/HtrA2 from the mitochondria to the cytosol enhanced MI/R injury in aging heart via promoting myocardial apoptosis. These studies may provide some therapies to prevent the over-release of HtrA2/Omi from mitochondria with aging and reduce the risk for MI/R in the elderly. This could help to explain the loss of ventricular function with age and may lead to discoveries of specific therapeutic interventions that can attenuate this type of cell loss (Wang et al., 2010).

\section{Prospect}

Aging has become a major health issue and socioeconomic burden worldwide. Coronary heart disease is the leading cause of death worldwide, for patients presented with an acute myocardial infarction, early and successful myocardial reperfusion is the most effective interventional strategy for reducing infarct size and improving clinical outcomes. The process of myocardial reperfusion itself, however, can induce injury to the myocardium, thereby reducing the beneficial effects of myocardial reperfusion. Aging renders the heart more susceptible to cell death from ischemia/reperfusion. In order to develop strategies aimed to limit reversible and irreversible myocardial damage in older patients, there is a need to better understand how aging increases myocardial apoptosis in myocardial ischaemia/reperfusion.

This chapter introduced that RNS signaling induced apoptosis contributes to increased susceptibility of aging hearts to myocardial ischemic injury, and the age-associated alterations in translocation of HtrA2/Omi from mitochondria to cytosol are implicated in the markedly increased risk for MI/R injury in old persons. 
As mentioned above, three RNS signaling pathways have been recognized .On the one hand, many studies show that RNS can be pro-apoptotic; on the other hand, many studies show that RNS exert anti-apoptotic effects through the same signaling pathway. Further studies should continue to elucidate the many factors that determine how RNS promotes or inhibits apoptosis.

Wang et al. results provide strong evidence that HtrA2/Omi plays a causative role in increased post-ischemic cardiomyocyte apoptosis in the aging heart, but the mechanisms of age-associated alterations in translocation of HtrA2/Omi from mitochondria to cytosol need elucidation.

There is an urgent need for more research of myocardial ischemia/reperfusion conducted on senescent animals. Some researches about RNS signaling induced apoptosis contributes to increased susceptibility of aging hearts to myocardial ischemic injury have been performed in rats or mice. However, biological signaling pathways, proteolytic portfolios, and the overall response to myocardial injury can be quite different in these small rodents when compared to larger mammals. While these murine studies have provided invaluable insight and provoked new hypotheses, they must be carried forward using large animals that more closely recapitulate the clinically-relevant context and for carefully designed clinical trials involving aged human subjects. There also needs to be better coordinated efforts between basic science investigators, clinical trial managers and physicians. (Spinale, 2010, as cited in Bujak et al., 2008; Singh et al., 2010; Lindsey, 2005; Juhaszova et al. ,2005 as cited in Bolli et al., 2004).

Otherwise, most studies have been conducted on healthy aging animals. Ischemic heart disease develops as a consequence of a number of etiological risk factors and always coexists with other disease states. These include systemic arterial hypertension and related left ventricular hypertrophy, hyperlipidemia, and atherosclerosis, diabetes and insulin resistance, as well as heart failure. These systemic diseases with aging as a modifying condition exert multiple biochemical effects on the heart that can potentially affect the development of I/R injury and interfere with responses to cardioprotective interventions. Therefore, the development of rational therapeutic approaches to protect the ischemic heart requires preclinical studies that examine cardioprotection specifically in relation to complicating disease states and risk factors. Surprisingly, relatively little effort has been made to uncover the cellular mechanisms by which risk factors and systemic diseases such as hypertension, hyperlipidemia and atherosclerosis, diabetes, insulin resistance, and heart failure interfere with cardioprotective mechanisms of aging (Ferdinandy et al., 2007).

Although, as mentioned above, RNS and HtrA2/Omi may be critical contributors in controlling apoptosis which determine the susceptibility of aging hearts to myocardial ischemic injury.

\section{References}

Althaus, J., Siegelin, M.D., Dehghani, F., Cilenti, L., Zervos, A.S. \& Rami A.(2007). The serine protease HtrA2/Omi is involved in XIAP cleavage and in neuronal cell death following focal cerebral ischemia/reperfusion. Neurochem int 50(1):172-180. 
Anathy, V., Aesif, S.W., Guala, A.S., Havermans, M., Reynaert, N.L., Ho, Y.S., Budd, R.C., and Janssen-Heininger, Y.M.W. (2009). Redox amplification of apoptosis by caspase-dependent cleavage of glutaredoxin 1 and S-glutathionylation of Fas. J Cell Biol 184(2):241-252.

Andoh, T., Chiueh, C.C. \& Chock, P. B.(2003). Cyclic GMP-dependent protein kinase regulates the expression of thioredoxin and thioredoxin peroxidase-1 during hormesis in response to oxidative stress-induced apoptosis. J Biol Chem. 278(2): 885-90.

Anversa, P., Palackal, T., Sonnenblick, E., Olivetti, G., Meggs, L., \& Capasso, J.M. (1990). Myocyte cell loss and myocyte cellular hyperplasia in the hypertrophied aging rat heart. Circulation Research 67(4): 871-885.

Beneke, S. \& Bürkle, A. (2007). Poly(ADP-ribosyl) ation in mammalian ageing, Nucleic Acids Res 35(22):7456-7465.

Benhar, M. \& Stamler, J.S. (2005). A central role for S-nitrosylation in apoptosis. Nat cell biol $7(7): 645-646$.

Bishopric, N.H. \& Webster, K.A. (2002). Preventing apoptosis with thioredoxin: ASK me how. Cir Res 90(12): 1237-1239.

Boyd, C.S. \& Cadenas, E. (2002). Nitric oxide and cell signaling pathways in mitochondrialdependent apoptosis. Biol chem 383(3-4):411-423.

Brüne, B. (2003). Nitric oxide: NO apoptosis or turning it ON? Cell Death Differ 10(8), 864869.

Calafell, R., Boada, J., Santidrian, A.F., Gil, J., Roig, T., Perales, J.C., and Bermudez, J. (2009). Fructose 1, 6-bisphosphate reduced TNF-[alpha]-induced apoptosis in galactosamine sensitized rat hepatocytes through activation of nitric oxide and cGMP production. Eur j pharmacol 610, 128-133.

Caretti, A., Bianciardi, P., Ronchi, R., Fantacci, M., Guazzi, M. \& Samaja, M. (2008). Phosphodiesterase-5 inhibition abolishes neuron apoptosis induced by chronic hypoxia independently of hypoxia-inducible factor-1 \{alpha\} signaling. Exp Biol Med 233(10):1222-1230.

Carr, A.C., McCall, M.R. \& Frei, B. (2000). Oxidation of LDL by myeloperoxidase and reactive nitrogen species: reaction pathways and antioxidant protection. Arteriosclerosis, thrombosis, and vascular biology 20:1716-1723.

Casagrande, S., Bonetto, V., Fratelli, M., Gianazza, E., Eberini, I., Massignan, T., Salmona, M., Chang, G., Holmgren, A. \& Ghezzi, P. (2002). Glutathionylation of human thioredoxin: a possible crosstalk between the glutathione and thioredoxin systems. Proc Natl Acad Sci USA 99(15):9745-9749.

Cassina, A.M., Hodara, R., Souza, J.M., Thomson, L., Castro, L., Ischiropoulos, H., Freeman, B.A. \& Radi, R. (2000). Cytochrome c nitration by peroxynitrite. J Biol Chem 275(28):21409-21415.

Chiche, J.D., Schlutsmeyer, S.M., Bloch, D.B., de la Monte, S.M., Roberts, J.D., Filippov, G., Janssens, S.P., Rosenzweig, A. \& Bloch, K.D. (1998). Adenovirus-mediated gene transfer of cGMP-dependent protein kinase increases the sensitivity of cultured vascular smooth muscle cells to the antiproliferative and pro-apoptotic effects of nitric oxide/cGMP. J Biol Chem 273(51): 34263-34271.

Choi, B.M., Pae, H.O., Jang, S.I., Kim, Y.M. \& Chung, H.T. (2002). Nitric oxide as a proapoptotic as well as anti-apoptotic modulator. J biochem mol biol 35(1):116-126. 
Ciani, E., Guidi, S., Della Valle, G., Perini, G., Bartesaghi, R. \& Contestabile, A. (2002). Nitric oxide protects neuroblastoma cells from apoptosis induced by serum deprivation through cAMP-response element-binding protein (CREB) activation. J Biol Chem 277(51), 49896-49902.

Ciriolo, M.R., De Martino, A., Lafavia, E., Rossi, L., Carr , M.T. \& Rotilio, G. (2000). Cu, Znsuperoxide dismutase-dependent apoptosis induced by nitric oxide in neuronal cells. J Biol Chemi 275(7):5065-5072.

De Nadai, C., Sestili, P., Cantoni, O., Li'.'vremont, J.P., Sciorati, C., Barsacchi, R., Moncada, S., Meldolesi, J. \& Clementi, E. (2000). Nitric oxide inhibits tumor necrosis factoralpha-induced apoptosis by reducing the generation of ceramide. Proc Nati Acad Sci USA 97(10), 5480-5485.

Desrois, M., Clarke, K., Lan, C., Dalmasso, C., Cole, M., Portha, B., Cozzone, P.J. \& Bernard, M. (2010). Upregulation of eNOS and unchanged energy metabolism in increased susceptibility of the aging type 2 diabetic GK rat heart to ischemic injury. Am J Physiol Heart Circ Physiol 299(5) :H1679-H1686.

Deveraux, Q.L., Leo, E., Stennicke, H.R., Welsh, K., Salvesen, G.S. \& Reed, J.C. (1999). Cleavage of human inhibitor of apoptosis protein XIAP results in fragments with distinct specificities for caspases. EMBO J. 18(19): 5242-5251.

Ferdinandy, P., Schulz, R. \& Baxter, G.F.,(2007). Interaction of cardiovascular risk factors with myocardial ischemia/reperfusion injury, preconditioning, and postconditioning. Pharmacol Rev 59(4): 418-58.

Francis, S.H., Busch, J.L., Corbin, J.D. \& Sibley, D. (2010). cGMP-dependent protein kinases and cGMP phosphodiesterases in nitric oxide and cGMP action. Pharmacol rev 62(3): 525-563.

Gao, F., Christopher, T.A., Lopez, B.L., Friedman, E., Cai, G. \& Ma, X.L. (2000). Mechanism of decreased adenosine protection in reperfusion injury of aging rats. Am J Physiol Heart Circ Physiol 279(1):H329-H338.

Gaston, B.M., Carver, J., Doctor, A. \& Palmer, L.A. (2003). S-nitrosylation signaling in cell biology. Mol interv 3(5): 253-263.

Giustarini, D., Rossi, R., Milzani, A., Colombo, R., and Dalle-Donne, I. (2004). SGlutathionylation: from redox regulation of protein functions to human diseases. Journal of cellular and molecular medicine 8, 201-212.

Gow, A.J., Farkouh, C.R., Munson, D.A., Posencheg, M.A. \& Ischiropoulos, H. (2004). Biological significance of nitric oxide-mediated protein modifications. Am J Physiol Lung Cell Mol Physiol 287(2): L262-L268.

Gu, Z., Kaul, M., Yan, B., Kridel, S.J., Cui, J., Strongin, A., Smith, J.W., Liddington, R.C. \& Lipton, S.A. (2002). S-nitrosylation of matrix metalloproteinases: signaling pathway to neuronal cell death. Science 297(5584): 1186-1190.

HA, K.S.O.O., KIM, K.I.M.O., KWON, Y.G., BAI, S.E.K., NAM, W.O.O.D., YOO, Y.M.I.N., KIM, P.K.M., CHUNG, H.U.N.T., BILLIAR, T.R., and KIM, Y.M. (2003). Nitric oxide prevents 6-hydroxydopamine-induced apoptosis in PC12 cells through cGMPdependent PI3 kinase/Akt activation. The FASEB journal 17, 1036.

Haendeler, J., Hoffmann, J., Tischler, V., Berk, B.C., Zeiher, A.M., \& Dimmeler, S. (2002). Redox regulatory and anti-apoptotic functions of thioredoxin depend on Snitrosylation at cysteine 69. Nat cell biol 4(10), 743-749. 
Haendeler, J., Hoffmann, J., Zeiher, A.M. \& Dimmeler, S. (2004). Antioxidant effects of Statins via S-nitrosylation and activation of thioredoxin in endothelial cells: a novel vasculoprotective function of Statins. Circulation. 110(7): 856-61.

Hara, M.R., Thomas, B., Cascio, M.B., Bae, B.I., Hester, L.D., Dawson, V.L., Dawson, T.M., Sawa, A. \& Snyder, S.H. (2006). Neuroprotection by pharmacologic blockade of the GAPDH death cascade. Proc Nati Acad Sci USA 103(10):3887-3889.

Hortelano, S., Alvarez, A.M. \& Boscá L. (1999). Nitric oxide induces tyrosine nitration and release of cytochrome c preceding an increase of mitochondrial transmembrane potential in macrophages. The FASEB J 13(15):2311-2317.

Hutton \& David. (2008). Older people in emergencies: considerations for action and policy development, In:World Health Organization. http://www.who.int/ageing/publications/Hutton_report_small.pdf

Jiang X., Kim H., Shu H., Zhao Y., Zhang H., Kofron J., Donnelly J., Burns D., Ng SC., Rosenberg S., Wang X. (2003). Distinctive roles of PHAP proteins and prothymosinalpha in a death regulatory pathway. Science 299(5604):223-226.

Jugdutt,B.I.\&Jelani,A.(2008).Aging and defective healing,adverse remodeling, and blunted post-conditioning in the reperfused wounded heart, J Am Coll Cardio 51(14): 13991403.

Juhaszova, M., Rabuel ,C., Zorov, D.B., Lakatta, E.G. \& Sollott ,SJ.(2005). Protection in the aged heart: preventing the heart-break of old age?, Cardiovasc Res 66(2): 233-244.

Kastrati, I., Edirisinghe, P.D., Wijewickrama, G.T. \& Thatcher, G.R. (2010). Estrogen-induced apoptosis of breast epithelial cells Is blocked by NO/cGMP and mediated by extranuclear estrogen receptors. Endocrinology 151(12):5602-5616.

Kim, S.J., Kuklov, A. \& Crystal, G.J. (2011) In vivo gene delivery of XIAP protects against myocardial apoptosis and infarction following ischemia/reperfusion in conscious rabbits. Life Sci. 88(13-14): 572-577.

Kim, Y.M., Chung, H.T., Kim, S.S., Han, J.A., Yoo, Y.M., Kim, K.M., Lee, G.H., Yun, H.Y., Green, A., Li, J., Simmons,R.L.\& Billiar,T.R. (1999). Nitric oxide protects PC12 cells from serum deprivation-induced apoptosis by cGMP-dependent inhibition of caspase signaling. J Neurosci 19(16): 6740-6747.

Kinsella, K. \& Velkoff, V. (2001). U.S. Census Bureau. An Aging World: 2001. U.S. Government Printing Office, Washington, DC.

Klatt, P. \& Lamas, S. (2000). Regulation of protein function by S-glutathiolation in response to oxidative and nitrosative stress. Eur J Biochem 267(16), 4928-4944.

Lakatta, E. G. \& Levy, D. (2003). Arterial and cardiac aging: major shareholders in cardiovascular disease enterprises: Part I: aging arteries: a "set up" for vascular disease. Circulation 107(1):139-146.

Lavrik, I.N., Golks, A. \& Krammer, P.H. (2005). Caspases: pharmacological manipulation of cell death. J Clin Invest 115(10):2665-2672.

Li, D., Qu, Y., Tao, L., Liu, H., Hu, A., Gao, F., Sharifi-Azad, S., Grunwald, Z., Ma, XL.and Sun JZ.(2006). Inhibition of iNOS protects the aging heart against beta-adrenergic receptor stimulation-induced cardiac dysfunction and myocardial ischemic injury. $J$ Surg Res. 131(1):64-72.

Li, S., Jiao, X., Tao, L., Liu, H., Cao, Y., Lopez, B.L., Christopher, T.A. \& Ma, X.L. (2007). Tumor necrosis factor-alpha in mechanic trauma plasma mediates cardiomyocyte apoptosis. Am JPhysiol Heart Circ Physiol 293(3):H1847-H852. 
Liang, F., Gao, E., Tao, L., Liu, H., Qu, Y., Christopher, T.A., Lopez, B.L. \& Ma, X.L. (2004). Critical timing of L-arginine treatment in post-ischemic myocardial apoptosis-role of NOS isoforms. Cardiovas res 62(3): 568-577.

Lincoln, D.T, Ali Emadi, E.M., Tonissen, K.F. \& Clarke, F.M.(2003). The thioredoxinthioredoxin reductase system: over-expression in human cancer. Anticancer Res 23(3B): 2425-2433.

Liu, H.R., Gao, E., Hu, A., Tao, L., Qu, Y., Most, P., Koch, W.J., Christopher, T.A., Lopez, B.L., Alnemri, E.S., Zervos, A.S., Ma, X.L. (2005). Role of HtrA2/Omi in Apoptotic Cell Death after Myocardial Ischemia and Reperfusion. Circulation 111(1):90-96.

Liu, P., Xu, B., Cavalieri, TA.\& Hock CE. (2002). Age-related difference in myocardial function and inflammation in a rat model of myocardial ischemia-reperfusion. Cardiovascular Research 56(3): 443-453.

Liu, Y. \& Min, W. (2002). Thioredoxin promotes ASK1 ubiquitination and degradation to inhibit ASK1-mediated apoptosis in a redox activity-independent manner. Circ Res 90(12): 1259-66.

MacDonald, Paul. (2002). Heart Disease, In: Encyclopedia.com, Encyclopedia of Aging, <http://www.encyclopedia.com/topic/Antilipemic_agents.aspx>

Mannick, J.B., Miao, X.Q. \& Stamler, J.S. (1997). Nitric oxide inhibits Fas-induced apoptosis. J Biol Chem 272(39):24125-24128.

Mannick, J.B. \& Schonhoff, C.M. (2004). NO means no and yes: regulation of cell signaling by protein nitrosylation. Free radic res 38(1):1-7.

Marshall, H.E. \& Stamler, J.S. (2002). Nitrosative stress-induced apoptosis through inhibition of NF-kappa B. J Biol Chem 277(37), 34223-34228.

Martin, S.J. (2002). Destabilizing influences in apoptosis: sowing the seeds of IAP destruction. Cell 109(7): 793-796.

Martinez-Ruiz, A., Cadenas, S. \& Lamas, S. (2011). Nitric oxide signaling: Classical, less classical, and nonclassical mechanisms. Free radic biol med 51(1):17-29.

Martins, L.M, Iaccarino, I., Tenev, T., Gschmeissner, S., Totty, N.F., Lemoine,N.R., Savopoulos, J., Gray, C.W., Creasy, C.L., Dingwall, C., Downward, J. The serine protease Omi/HtrA2 regulates apoptosis by binding XIAP through a reaper-like motif. J Biol Chem 277(1):439-444.

Mathewson, F. \& Varnam, G. (1960). Abnormal electrocardiograms in apparently healthy people. I. Long term follow-up study. Circulation 21(2):196-203.

Mitchell, D.A. \& Marletta, M. A.(2005). Thioredoxin catalyzes the S-nitrosation of the caspase-3 active site cysteine. Nat Chem Biol 1(3): 154-158.

Morisaki,N.,Saito,I.,Tamura, K.,Tashiro, J.,Masuda, M..,Kanzaki, T.,Watanabe, S., Masuda, Y., Saito, Y.(1997). New indices of ischemic heart disease and aging:studies on the serum levels of soluble intercellular adhesion molecule-1 (ICAM-1) and soluble vascular cell adhesion molecule-1 (VCAM-1) in patients with hypercholesterolemia and ischemic heart disease. Atherosclerosis 131(1) :43-48.

Nakagawa, H., Komai, N., Takusagawa, M., Miura, Y., Toda, T., Miyata, N., Ozawa, T., Ikota ,N.(2007). Nitration of specific tyrosine residues of cytochrome $\mathrm{C}$ is associated with caspase-cascade inactivation. Biol Pharm Bull 30(1):15-20.

Ohshima S. (2006). Apoptosis and necrosis in senescent human fibroblasts. Annals of the New York Academy of Sciences 1067:228-234. 
Okada, H., Suh, W.K., Jin, J., Woo, M., Du, C., Elia, A., Duncan, G.S., Wakeham, A., Itie, A., Lowe, S.W., Wang, X. \& Mak, T.W. (2002). Generation and characterization of Smac/DIABLO-deficient mice. Mol Cell Biol 22(10): 3509-3517.

Olivetti, G., Melissari, M., Capasso, J. \& Anversa, P. (1991). Cardiomyopathy of the aging human heart. Myocyte loss and reactive cellular hypertrophy. Circulation Research 68(6): 1560-1568.

Park, H.S., Yu, J.W., Cho, J.H., Kim, M.S., Huh, S.H., Ryoo, K., \& Choi, E.J. (2004). Inhibition of apoptosis signal-regulating kinase 1 by nitric oxide through a thiol redox mechanism. J Biol Chem 279(9), 7584-7590.

Park,J.B., Park,I.C., Park,S.J., Jin,H.O., Lee,J.K., \& Riew, K.D.(2006). Anti-apoptotic effects of caspase inhibitors on rat intervertebral disc cells. J Bone Joint Surg Am 88(4):771779 .

Patel, R.P., McAndrew, J., Sellak, H., White, C.R., Jo, H., Freeman, B.A. \& Darley-Usmar, V.M. (1999). Biological aspects of reactive nitrogen species. Biochimica et Biophysica Acta (BBA)-Bioenergetics 1411(2-3):385-400.

Peluffo, G. \& Radi, R. (2007). Biochemistry of protein tyrosine nitration in cardiovascular pathology. Cardiovas res 75(2):291-302.

Phaneuf ,S. \& Leeuwenburg, C. (2002). Cytochrome c release from mitochondria in the aging heart : a possible mechanism for apoptosis with age. Am J Physiol Regulatory Integrative Comp Physiol 282(2): R423-R430.

Qanungo, S., Starke, D.W., Pai, H.V., Mieyal, J.J. \& Nieminen, A.L. (2007). Glutathione supplementation potentiates hypoxic apoptosis by S-glutathionylation of p65-NFkappa B. J Biol Chem 282(25):18427-18436.

Reinehr R., Görg B., Höngen A., and Häussinger D. (2004). CD95-tyrosine nitration inhibits hyperosmotic and CD95 ligand-induced CD95 activation in rat hepatocytes. J Biol Chem 279(11): 10364-10373.

Rus, A., del Moral, M.L., Molina, F. \& Peinado, M.A. (2010). Does inducible NOS have a protective role against hypoxia/reoxygenation injury in rat heart? Cardiovas Pathol 20(1):e17-25.

Salvesen, G. S. \& Duckett, C. S. (2002). IAP proteins: blocking the road to death's door. Nat. Rev. Mol. Cell Biol 3:401-410.

Schonhoff, C.M., Gaston, B. \& Mannick, J.B. (2003). Nitrosylation of cytochrome c during apoptosis. J Biol Chem 278(20), 18265-18270.

Schonhoff, C.M., Matsuoka, M., Tummala, H., Johnson, M.A., Estevéz A.G., Wu, R., Kamaid, A., Ricart, K.C., Hashimoto, Y., Gaston, B., Macdonald,T.L., Xu, Z. \& Mannick, J.B. (2006). S-nitrosothiol depletion in amyotrophic lateral sclerosis. Proc Nati Acad Sci USA 103(7): 2404-2409.

Shi, Y. A conserved tetrapeptide motif: potentiating apoptosis through IAP-binding.(2002). Cell Death Differ 9(2), 93-95.

Spinale,F.G. (2010).Amplified bioactive signaling and proteolytic enzymes following ischemia reperfusion and aging: remodeling pathways that are not like a fine wine. Circulation 122(4):322-324.

Srinivasula, S.M., Gupta, S., Datta, P., Zhang, Z., Hegde, R., Cheong,N., Fernandes-Alnemri, T. \& Alnemri,E.S. (2003). Inhibitor of Apoptosis Proteins Are Substrates for the Mitochondrial Serine Protease Omi/HtrA2. J biol chem 278(34): 31469-31472. 
Stamler, J.S., Lamas, S. \& Fang, F.C. (2001). Nitrosylation. the prototypic redox-based signaling mechanism. Cell 106(6): 675-683.

Suzuki Y, Lmai Y, Nakayama H, Takahashi K, Koji Takio, Ryosuke Takahashi. A serine protease, HtrA2, is released from the mitochondria and interacts with XIAP, inducing cell death. Mol cell 2001; 8:613-621.

Török, N.J., Higuchi, H., Bronk, S. \& Gores, G.J. (2002). Nitric oxide inhibits apoptosis downstream of cytochrome $C$ release by nitrosylating caspase 9 . Cancer res 62(6), 1648-1653.

Taimor, G., Hofstaetter, B. \& Piper, H. M.(2000). Apoptosis induction by nitric oxide in adult cardiomyocytes via cGMP-signaling and its impairment after simulated ischemia. Cardiovasc res 45(3):588-594.

Tao, L., Jiao, X., Gao, E., Lau, W., Yuan, Y., Lopez, B., Christopher, T., Ramachandrarao, S.P., Williams, W., Southan, G., Sharma, K., Koch, W. \& Ma, X. (2006). Nitrative inactivation of thioredoxin-1 and its role in postischemic myocardial apoptosis. Circulation. 114(13): 1395-1402.

Taylor, C.T. \& Moncada, S. (2010). Nitric oxide, cytochrome C oxidase, and the cellular response to hypoxia. Arterioscler Thromb Vascular Biol 30(4):643-647.

U.S. Census Bureau. International database. Table 094. Midyear population, by age and sex. Available at http://www.census.gov/population/www/projections/ natdet-D1A. html.

Vandervliet, A., Eiserich, J.P., O'Neill, C.A., Halliwell, B. \& Cross, C.E. (1995). Tyrosine modification by reactive nitrogen species: a closer look. Arch biochem biophys 319(2):341-349.

Wang, K., Zhang, J, Tian., J, Liu, J., Wang, L., Ma, X., Guo, L., Yang, G., Liu, H. (2008). The pro-apoptotic effect of HtrA2/Omi in myocardial ischemia/ reperfusion injury in aged rats. Acta Physiology Sinica. 60 (Suppl.1):195-300.

Wang, K., Liu, J., Tian., J., Yan, Z., Zuo, L., Zheng, R. Liu, H. (2010). Time course of XIAP expression after myocardial ischemia/reperfusion in adult rats. Chinese Journal of Cardiovascular Review 8(5):376-379.

Wu, G., Chai, J., Suber, T.L.,Wu, J.W., Du ,C., Wang, X. \& Shi, Y. (2000). Structural basis of IAP recognition by Smac/DIABLO. Nature. 408(6815): 1008-1012.

Wu, K., Jiang, L., Cao, J., Yang, G., Geng, C. \& Zhong, L. (2007). Genotoxic effect and nitrative DNA damage in HepG2 cells exposed to aristolochic acid. Mutation Research/Genetic Toxicology and Environmental Mutagenesis 630(1-2):97-102.

Yellon, D.M. \& Hausenloy, D.J.(2007). Myocardial reperfusion injury, N Engl J Med .357(11): :1121-1135.

Yuyama, K., Yamamoto, H., Nishizaki, I., Kato, T., Sora, I. \& Yamamoto, T. (2003). Caspaseindependent cell death by low concentrations of nitric oxide in PC12 cells: involvement of cytochrome $\mathrm{C}$ oxidase inhibition and the production of reactive oxygen species in mitochondria. J neurosci res 73,(3):351-363.

Zhang, J., Jin, B., Li, L., Block, E.R. \& Patel, J.M. (2005). Nitric oxide-induced persistent inhibition and nitrosylation of active site cysteine residues of mitochondrial cytochrome-c oxidase in lung endothelial cells. Am J Physiol Cell Physiol 288(4):C840-C849.

Zhang, JH., Zhang,Y., Herman B.(2003). Caspases, apoptosis and aging. Aging Research Review 2(4):357-366. 
Zhang, H., Tao, L., Jiao, X., Gao, E., Lopez, B. L., Christopher, T.A., Koch, W. \& Ma ,X.L. (2007). Nitrative thioredoxin inactivation as a cause of enhanced myocardial ischemia/reperfusion injury in the aging heart. Free Radical Biology and Medicine 43(1):39-47. 


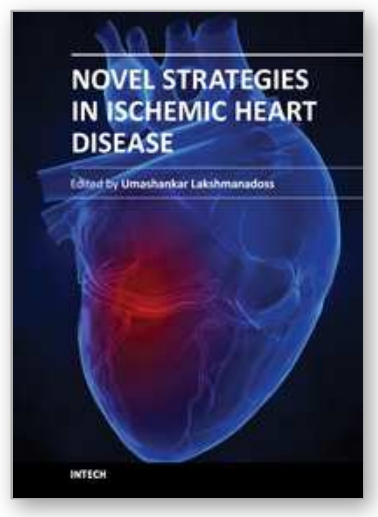

\author{
Novel Strategies in Ischemic Heart Disease \\ Edited by Dr. Umashankar Lakshmanadoss
}

ISBN 978-953-51-0184-0

Hard cover, 450 pages

Publisher InTech

Published online 29, February, 2012

Published in print edition February, 2012

The first edition of this book will provide a comprehensive overview of ischemic heart disease, including epidemiology, risk factors, pathogenesis, clinical presentation, diagnostic tests, differential diagnosis, treatment, complications and prognosis. Also discussed are current treatment options, protocols and diagnostic procedures, as well as the latest advances in the field. The book will serve as a cutting-edge point of reference for the basic or clinical researcher, and any clinician involved in the diagnosis and management of ischemic heart disease. This book is essentially designed to fill the vital gap existing between these practices, to provide a textbook that is substantial and readable, compact and reasonably comprehensive, and to provide an excellent blend of "basics to bedside and beyond" in the field of ischemic heart disease. The book also covers the future novel treatment strategies, focusing on the basic scientific and clinical aspects of the diagnosis and management of ischemic heart disease.

\title{
How to reference
}

In order to correctly reference this scholarly work, feel free to copy and paste the following:

Huirong Liu, Ke Wang, Xiaoliang Wang, Jue Tian, Jianqin Jiao, Kehua Bai, Jie Yang and Haibo Xu (2012). Aging, Reactive Nitrogen Species and Myocardial Apoptosis Induced by Ischemia/Reperfusion Injury, Novel Strategies in Ischemic Heart Disease, Dr. Umashankar Lakshmanadoss (Ed.), ISBN: 978-953-51-0184-0, InTech, Available from: http://www.intechopen.com/books/novel-strategies-in-ischemic-heart-disease/agingreactive-nitrogen-species-and-myocardial-apoptosis-induced-by-ischemia-reperfusion-injury

\section{INTECH}

open science | open minds

\section{InTech Europe}

University Campus STeP Ri

Slavka Krautzeka 83/A

51000 Rijeka, Croatia

Phone: +385 (51) 770447

Fax: +385 (51) 686166

www.intechopen.com

\section{InTech China}

Unit 405, Office Block, Hotel Equatorial Shanghai

No.65, Yan An Road (West), Shanghai, 200040, China

中国上海市延安西路65号上海国际贵都大饭店办公楼405单元

Phone: +86-21-62489820

Fax: $+86-21-62489821$ 
(C) 2012 The Author(s). Licensee IntechOpen. This is an open access article distributed under the terms of the Creative Commons Attribution 3.0 License, which permits unrestricted use, distribution, and reproduction in any medium, provided the original work is properly cited. 